\title{
Fluctuating Electric Field and Franz-Keldysh Effect inside an Acoustoelectric Domain
}

\author{
W. WONNEBERGER \\ Physikalisches Institut der Universität Freiburg i. Br. \\ (Z. Naturforsch. 27 a, 956-960 [1972] ; received 15 March 1972)
}

\begin{abstract}
The mean squared electric field $\left\langle\mathfrak{F}^{2}\right\rangle$ associated with the great number of vibrational waves excited in an acoustoelectric domain is determined by means of an asymptotic analysis of the current saturation parameter. It is found that the Franz-Keldysh effect caused by $\left\langle\mathfrak{F}^{2}\right\rangle$ can account for the optical transmission modulation observed during the passage of an acoustoelectric domain.
\end{abstract}

\section{Introduction}

Acoustoelectric domains in piezoelectric semiconductors are associated with a large number of effects. Among them, the optical transmission modulation during the passage of the domain has been investigated in recent years by several authors ${ }^{1-6}$. It was found that the transmission modulation is most pronounced near the absorption edge of the unperturbed semiconductor.

Among a variety of other possibilities the FranzKeldysh effect ${ }^{7,8}$ has been taken into consideration as the cause of the observed transmission modulation. From known values of the dc electric field inside an acoustoelectric domain and some more sophisticated arguments (cf. ${ }^{1-6}$ ) one is able to rule out the dc electric field as the principal cause of the transmission modulation. What remains to be considered more closely is the fluctuating electric field associated with acoustic flux in_a piezoelectric semiconductor.

In the earlier literature it is commonly doubted that the many vibrational modes excited in an acoustoelectric domain are able to produce a fluctuating electric field of the required strength. However, KOHN and LAMPERT ${ }^{5}$ have recently substantiated the latter idea. Their reasoning proceeds via a detailed analysis of their own experimental results, excluding all but the above mentioned case. The authors are not able, however, to determine the fluctuating electric field and to directly establish the connection with the Franz-Keldysh effect. Therefore it seems worthwhile, to apply a theory of nonlinear ultrasound amplification to calculate the fluctuating electric field inside an acoustoelectric do-

Reprint requests to Dr. W. Wonneberger, Physikalisches Institut der Universität Freiburg, D-7800 Freiburg $i$. Br., Hermann-Herder-Straße 3. main and to compare the theoretical predictions with the experimental results.

In the second section we investigate the nature of the fluctuating electric field $\mathbb{E}$. In Section 3 we establish the relation of the mean squared electric field $\left\langle\mathfrak{F}^{2}\right\rangle$ to the current saturation parameter. We then study the current saturation parameter $f$ and its asymptotic behaviour appropriate for high flux acoustoelectric domains. From a knowledge of $f$ and of the mean wave vector $\overline{\boldsymbol{Q}}$ of the amplified vibrational flux, the value of $\left\langle\overleftarrow{\digamma}^{2}\right\rangle$ is easily obtained. In Section 4 we evaluate our results numerically and compare them with published experiments.

\section{The Fluctuating Electric Field}

In an acoustoelectric domain each of the many excited vibrational modes is accompanied by an electric ac field $\widetilde{\mathfrak{F}}(\boldsymbol{x}, t) \exp \{-i \omega(\boldsymbol{x}) t\}$. Here, $\boldsymbol{x}$ is the wave vector and $\omega(\boldsymbol{x})$ is the frequency of the mode. The fields $\widetilde{F}(\boldsymbol{x}, t)$ should not be regarded as a mere by-product of the acoustic flux. They are precisely the reason for the electronic system to react on the phonon system as a loss or gain mechanism depending on whether the sound velocity $v_{\text {s }}$ exceeds the electronic drift velocity or vice versa. Therefore one may consider the ac electric fields as the basic quantities in the theory of acoustoelectric phenomena. The microscopic ac fields give rise to an instantaneous total field

$\mathfrak{F}(\boldsymbol{r}, t)=\sum_{\boldsymbol{x}} \widetilde{\mathfrak{F}}(\boldsymbol{x}, t) \exp \{i \boldsymbol{x} \cdot \boldsymbol{r}-i \omega(\boldsymbol{x}) t\}$.

$\mathcal{F}(\boldsymbol{r}, t)$ is an extremely complicated function of position and time. In fact, one should regard $F$ as a stochastic variable and describe its properties in terms of statistical averages. 
In multimode theories of nonlinear ultrasound amplification ${ }^{9-13}$ one frequently employes the random phase approximation (RPA). In this case $\mathbb{E}$ has zero mean: $\langle \&\rangle_{\mathrm{RPA}}=0$. Furthermore

$$
\left\langle\mathscr{F}^{2}\right\rangle_{\mathrm{RPA}}=\sum_{x}\left\langle|\tilde{F}(\boldsymbol{x}, t)|^{2}\right\rangle \text {. }
$$

If one has reasons to believe that in a specific experiment the average \langle\rangle$_{\mathrm{RPA}}$ may replace the averaging automatically involved in the experiment, one may conclude that physical effects depending linearly on the electric field are not affected by the fluctuating field F. In contrast to this, a quadratic effect can be quite pronounced if a very great number of electric fields are involved in $(5$.

We do not enter into a detailed discussion of the delicate distinction between $\overline{\widetilde{F}^{2}}$ and $\left\langle\mathfrak{F}^{2}\right\rangle_{\mathrm{RPA}}$ since the difference between both is expected to be small for the case considered here ${ }^{*}$.

We instead turn to a determination of the quantity $\left\langle\mathbb{F}^{2}\right\rangle_{\mathrm{RPA}}$. In order to make the problem tractable we assume that the modes involved in $F$ are concentrated in a small frequency band whose frequency spread $\Delta \omega$ is smaller than the mean frequency $\bar{\omega}$. This is a reasonable assumption for fully developed acoustoelectric domains (cf. e. g. ${ }^{6,15,16}$ ).

\section{The Current Saturation Parameter}

From the above remarks about the role of the ac electric fields it is clear that they manifest themselves most directly through the current saturation parameter $f$ defined as

$$
f=-j_{\mathrm{dc}}^{\mathrm{ae}} /\left(j_{\mathrm{dc}}^{\Omega}-j_{\mathrm{dc}}^{\mathrm{synch}}\right) .
$$

Here, $j_{\mathrm{dc}}^{\mathrm{ae}}$ is the dc acoustoelectric current density. $j$ dc the fictitious ohmic current density corresponding to the dc electric field $E_{\mathrm{dc}}^{\text {in }}$ inside the domain:

$$
j_{\mathrm{dc}}^{\Omega}=e n_{0} \mu E_{\mathrm{dc}}^{\mathrm{in}}
$$

and $j{ }_{\mathrm{dc}}^{\mathrm{sunch}}$ is given by

$$
j_{\mathrm{dc}}^{\mathrm{synch}}=e n_{0} v_{\mathrm{8}} .
$$

The true current density $j_{\text {dc }}$ through the sample is

$$
j_{\mathrm{dc}}=e n_{0} \mu E_{\mathrm{dc}}^{\text {out }}=j_{\mathrm{dc}}^{\Omega}+j_{\mathrm{dc}}^{\mathrm{ae}} .
$$

* In photon-counting experiments the situation is more com plex due to the strongly nonlinear dependence of the counting distribution on a squared random variable. In fact, one has found evidence from photon-counting measurements ${ }^{14}$ that an incoherent superposition of modes like in Eq. (2) is violated for the acoustic displacement fields if a time average shorter than the coherence times of the individual modes is performed.
Equation (3) gives for $j_{\mathrm{dc}}$

$$
j_{\mathrm{dc}}=f j_{\mathrm{dc}}^{\mathrm{synch}}+(1-f) j_{\mathrm{dc}}^{\Omega} .
$$

$f$ can therefore be identified with the dynamic trapping factor $p$ appearing in the work of MOORE ${ }^{17}$.

For practical calculations another writing of Eq.

(3) is useful, namely

$$
f=1-\frac{\mu E_{\mathrm{dc}}^{\text {out }} / v_{\mathrm{s}}-1}{\mu E_{\mathrm{dc}}^{\text {in }} / v_{\mathrm{s}}-1}=1-\frac{\gamma^{\text {out }}}{\gamma^{\text {in }}} .
$$

The current saturation parameter $f$ may be determined numerically for any specific experiment. One then needs analytic formulae relating $f$ to the domain parameters including the fluctuating electric field. For the one dimensional and single mode case such relations have been given in Ref. ${ }^{18}$ and Ref. ${ }^{19}$. They are certainly valid in high flux situations. Furthermore, the theory ${ }^{19}$ which is particular appropriate for practical calculations has been extended to the multimode case described above ${ }^{13} . f$ is then given by

$$
f=\frac{2 y}{(-\chi)} \operatorname{Re} \frac{J_{1-i \chi}(2 i y)}{J_{-i \chi}(2 i y)} .
$$

To obtain Eq. (4) the explicite expression for the nonlinear response function $\varepsilon_{\mathrm{nl}}{ }^{20}$ must be inserted in the relation Eq. (27) of Ref. ${ }^{19}$.

In Eq. (4) the activity parameter $\chi$ is given by $\chi=\left(1-\mu E_{\mathrm{dc}}^{\mathrm{in}} / v_{\mathrm{s}}\right) \omega_{\mathrm{D}} / \bar{\omega}, \omega_{\mathrm{D}}$ is the diffusion fre. quency, $\bar{\omega}$ the mean frequency of the acoustic flux, $2 y$ is the bunching parameter which is related to the piezoelectric potential amplitudes $2 y_{\omega}$ (in units of $k_{\mathrm{B}} T$ ) of the individual modes by

$$
(2 y)^{2}=\sum_{\omega \approx \bar{\approx}}\left(2 y_{\omega}\right)^{2} .
$$

This equation is quite analogous to Eq. (2) besides referring to the potentials instead to the fields and containing positive frequency parts only. Therefore, one finally has

$$
\overline{\mathfrak{F}^{2}} \triangleq\left\langle\mathfrak{F}^{2}\right\rangle_{\mathrm{RPA}}=2\left(\frac{y k_{\mathrm{B}} T \bar{Q}_{-}}{e}\right)^{2} .
$$

Though the relation (4) establishes a one to one correspondence between $f$ and $2 y$ for each fixed value of $\chi$ it cannot be applied directly because of its functional complexity. Furthermore, high flux acoustoelectric domains pose a special problem: The values of $f$ are very near to one. The difference $1-f$ frequently is of the order $10^{-2}$ or smaller. Therefore the numerical plots given by TIEN ${ }^{21}$ and the 
almost identical results of Ref. ${ }^{19}$ derived from Eq. (4) cannot be used. One has to resort to asymptotic expansions. But it is hopeless, to use asymptotic expansions of Bessel-functions in order to evaluate the Bessel-function quotient in Equation (4). These expansions are much too inaccurate for our problem. The quotient of Bessel-functions in Eq. (4) may, however, be expressed as the continued fraction:

$$
\frac{J_{1-i \chi}(2 i y)}{J_{-i \chi}(2 i y)}=\frac{i y \mid}{\mid 1-i \chi}+\frac{y^{2} \mid}{2-i \chi}+\frac{y^{2} \mid}{\mid 3-i \chi}+\ldots
$$

which is much more at the heart of the mathematical problems involved in the theory of nonlinear ultrasound amplification. For instance, one sees immediately that for $2 y>|\chi|>1$ the number $n$ of harmonics essentially excited in the electronic density fluctuations is given by $n \approx y \gg 1$.

In high flux acoustoelectric domains, one usually has $|\chi| \gg 1$. This will enable us to evaluate Eq. (4) asymptotically. For this purpose we write Eq. (7) approximately $(|\chi| \gg 1,2 y \gtrless|\chi|$ but not $2 y \approx|\chi|)$ as:

$z=\frac{J_{1-i \chi}(2 i y)}{J_{-i \chi}(2 i y)}=\frac{i y}{\mid 1-i \chi}+\frac{y^{2}}{1-i \chi}+\frac{y^{2} \mid}{2-i \chi}+\ldots$

from which one obtains

$$
z=i y /(1-i \chi-i y z)
$$

with the solution

$$
z=-i \frac{1-i \chi}{2 y}+i \sqrt{1+\left(\frac{1-i \chi}{2 y}\right)^{2}} .
$$

For $2 y>|\chi|$ this equation is easily evaluated to yield the following expression for the current satu. ration parameter $f$

$$
\begin{aligned}
f=1-\frac{1}{2 y}[1 & +\frac{1}{2}\left(\frac{\chi}{2 y}\right)^{2} \\
& \left.+\frac{3}{8}\left(\frac{\chi}{2 y}\right)^{4}+\frac{5}{16}\left(\frac{\chi}{2 y}\right)^{6}+\ldots\right] .
\end{aligned}
$$

To a good approximation, one finally gets for the bunching parameter $2 y$

$$
2 y=\frac{1}{1-f}+\frac{1}{2} \chi^{2}(1-f)+O\left(\chi^{4}(1-f)^{3}\right) .
$$

Note, that the simple relation

$$
2 y=1 /(1-f)
$$

gives a lower bound for $2 y$ which is already correct within $15 \%$ for $2 y>2|\chi|$.

\section{Comparison with Experiments}

Apart from material constants like sound velocity, diffusion frequency and resistivity of the piezoelectric crystals the following "dynamical" quantities must be known for each individual experiment:

1. drift velocity of "free" carriers inside the domain (in units of $v_{\mathrm{s}}$ ),

2. drift velocity of carriers outside the domain (in units of $v_{\mathrm{s}}$ ), and

3. mean frequency $\bar{v}$ of the acoustic flux.

Unfortunately, these acoustoelectric data are not specified completely in the works ${ }^{1-6}$. We therefore must collect the missing data from related work on high flux acoustoelectric domains.

In our first example we refer to the recent experimental work of KOHN and LAMPERT ${ }^{5}$ on CdS at room temperature. In $\mathrm{CdS}, E_{\mathrm{dc}}^{\text {out }}$ is only slightly above the synchroneous field. Therefore the outer criticallity $\gamma^{\text {out }}=\left(\mu E_{\mathrm{dc}}^{\text {out }} / v_{\mathrm{s}}\right)-1$ is a very delicate quantity from an experimental point of view. Note, that $\gamma^{\text {out }}$ does not only depend on specimen data but also reflects the dynamical state inside the domain. From the work of HAYDL et al. ${ }^{22}$ one can conclude that a value of about $10 \%$ for $\gamma^{\text {out }}$ is a reasonable estimate for the CdS data compiled in Table 1 . Using Eq. (9') which as a lower bound to the bunching parameter is very appropriate for our estimates, then gives the values for $2 y$. In the next step we need the mean frequency $\bar{v}$. We determine $\bar{v}$ as the frequency $\tilde{\boldsymbol{v}}_{\mathrm{m}}$ of maximum nonlinear gain from the relation $\tilde{\nu}_{\mathrm{m}}=\nu_{\mathrm{m}}(y)^{-1 / 2}$ valid for $|\chi|<2 y \gg 1$ (Ref. ${ }^{23}$, cf. also Ref. ${ }^{24}$ ) ${ }^{* *}$. All other data are extracted from the literature cited in Table 1 . As the result we obtain from Eq. (6) the correct value for $\left\langle\xi^{2}\right\rangle$ of about $1 \cdot 10^{10}\left(\mathrm{~V} \mathrm{~cm}^{-1}\right)^{2}$ needed to explain the observations in Reference ${ }^{5}$.

Our second example refers to the work of SPEARS and $\mathrm{BRAY}{ }^{2,25}$ on GaAs at the temperature of liquid nitrogen. Here, the data are almost complete except for the mean frequency $\bar{v}$. We have determined $\bar{\nu}$ in the same way as for CdS. The result for $\left\langle\zeta^{2}\right\rangle$ is again in good agreement with the required value of $1 \cdot 10^{9} \mathrm{~V}^{2} \mathrm{~cm}^{-2}$ estimated from direct measurements of the Franz-Keldysh effect in $\mathrm{GaAs}^{26}$.

* The weak flux situation is characterized by $|\chi| \approx 2 y^{23}$. For strong flux domains, this condition is modified to $2 y>|\chi|$. 
Table 1. Survey of data for two specific experiments ${ }^{2,5}$ on optical transmission modulation by an acoustoelectric domain.

\begin{tabular}{|c|c|c|c|c|c|}
\hline Crystal & & CdS & $\begin{array}{l}\text { Ref. } \\
5\end{array}$ & GaAs & $\underset{\mathbf{2}}{\text { Ref. }}$ \\
\hline Temperature $T$ & $\left({ }^{\circ} \mathrm{K}\right)$ & 300 & 5 & 77 & 2 \\
\hline Resistivity $x$ & $(\Omega \mathrm{cm})$ & 10 & 5 & 0.08 & 2 \\
\hline Drift mobility $\mu$ & $\left(\mathrm{cm}^{2} \mathrm{~V}^{-1} \mathrm{~s}^{-1}\right)$ & $2.5 \cdot 10^{2}$ & & $8 \cdot 10^{3}$ & 2 \\
\hline Frequency $\omega_{m}$ of $\max$. lin. electr. gain & $\left(\mathrm{s}^{-1}\right)$ & $2.4 \cdot 10^{10}$ & & $1.6 \cdot 10^{11}$ & \\
\hline Diffusion frequency $\omega_{\mathrm{D}}$ & $\left(s^{-1}\right)$ & $4.7 \cdot 10^{9}$ & & $2.1 \cdot 10^{9}$ & \\
\hline Domain field $E_{\mathrm{dc}}^{\text {in }}$ & $\left(\mathrm{V} \mathrm{cm}^{-1}\right)$ & $5 \cdot 10^{4}$ & 5 & $5 \cdot 10^{3}$ & 2,25 \\
\hline Inner criticallity $\gamma^{\text {in }}$ & & 70 & & 120 & \\
\hline Outer criticallity $\gamma^{\text {out }}$ & & 0.1 & 22 & 1 & 2 \\
\hline Current saturation parameter $f$ & & $1-1 / 700$ & & $1-1 / 120$ & \\
\hline Bunching parameter $2 y$ & & 700 & & 120 & \\
\hline Mean frequency $\bar{\nu}$ & $(\mathrm{Hz})$ & $2 \cdot 10^{8}$ & & $3.3 \cdot 10^{9}$ & \\
\hline Mean wave vector $\bar{Q}$ & $\left(\mathrm{~cm}^{-1}\right)$ & $7.3 \cdot 10^{3}$ & & $6.2 \cdot 10^{4}$ & \\
\hline Activity parameter $\chi$ & & -260 & & -12 & \\
\hline Mean squared electric field $\left\langle\mathfrak{F}^{2}\right\rangle_{\text {theor. }}$ & $\left(\mathrm{V}^{2} \mathrm{~cm}^{-2}\right)$ & $9 \cdot 10^{9}$ & & $1.2 \cdot 10^{9}$ & \\
\hline Mean squared electric field $\left\langle\mathfrak{G}^{2}\right\rangle$ exp. & $\left(\mathrm{V}^{2} \mathrm{~cm}^{-2}\right)$ & $1 \cdot 10^{10}$ & 5 & $1.0 \cdot 10^{9}$ & 2,26 \\
\hline
\end{tabular}

\section{Discussion}

Within the random phase approximation and for acoustic flux concentrated in a small frequency band, we have determined the strength $\left\langle\mathbb{F}^{2}\right\rangle$ of the fluctuating electric field $\&$ inside a high flux acoustoelectric domain.

Though the numerical results of Section 4 are in accord with the experimental estimates for $\left\langle\mathfrak{F}^{2}\right\rangle$ some uncertainty remains about the values of the outer criticallity and the mean frequency of the amplified acoustic flux which played an essential role in the above analysis. The first problem requires very careful experimental examination involving new measuring techniques. The second problem has been solved since years for instance by the Brillouin scattering method 16, 27, 28 but not in conjunction with measurements of the transmission modulation. An exception is the very recent experimental work ${ }^{6}$ on CdS which indeed shows that acoustic flux of rather low frequencies is involved. It is not clear, however, that our procedure to take the frequency of maximum nonlinear gain as $\bar{\nu}$ is strictly correct.

1 C. S. Kumar, P. O. Sliva, and R. Bray, Phys. Rev. 169, 680 [1968].

2 D. L. Spears and R. Bray, Appl. Phys. Letters 12, 118 [1968].

3 C. S. Kumar and W. G. Hutchinson, J. Appl. Phys. 40, 4687 [1969].

4 K. Yamamoto, M. Yamada, and K. Abe, J. Appl. Phys. 41, 450 [1970].

5 E. S. Kohn and M. A. Lampert, Phys. Rev. B 4, 4479 [1971].

6 K. Yamamoto, F. Murai, and K. Abe, Sol. State Comm. 10, 273 [1972]

7 W. FranZ, Z. Naturforsch. 13 a, 484 [1958].
We believe, that the downward shift of the frequency of peak acoustic intensity observed in acoustoelectric domains is governed by the shifting frequencies of maximum nonlinear gain. But in a high flux domain the gain has saturated so much and the gain curve has become so flat as function of frequency that the noise redistribution might remain behind the downward shift of the frequency of maximum nonlinear gain.

One may forget about these problems if the relevant data are known from experiments. The formulae of Section 3 then provide a convenient way to relate the important dynamical domain variables to one another. These are the current saturation parameter, the activity parameter, and the bunching parameter, the latter being almost a synonym for the mean squared fluctuating electric field inside an acoustoelectric domain.

\section{Acknowledgement}

Helpful discussions with Prof. H. G. Rerk, Dr. W. H. HAYDL, and Dr. M. Schulz are gratefully acknowledged.

8 L. V. Keldysh, Zh. Eksperim. i. Teor. Fiz. 34, 1138 [1958]; (Sov. Phys. JETP 7, 788 [1958]).

9 K. Yamada, Phys. Rev. 169, 690 [1968].

10 V. L. Gurevich, V.D. Kagan, and B. D. Laikhtman, Sov. Phys. JETP 27, 102 [1968].

11 P. N. Butcher and N. R. Ogg, Phys. Letters 30 A, 66 [1969].

12 P. N. Butcher and J. Slechta, Brit. J. Phys. C 4, 870 [1971].

13 W. WONNEBERger, Z. Naturforsch. 26 a, 1625 [1971].

14 F. Siebert and W. Wonneberger, Phys. Letters 37 A, 367 [1971]. 
15 M. Bruun, W. Wettiling, and N. I. Meyer, Phys. Letters 31 A, 31 [1970].

16 D. L. Spears, Phys. Rev. B 2, 1931 [1970].

17 A. Moore, J. Appl. Phys. 38, 2327 [1967].

18 R. K. L. Gay and H. L. Hartnagel, Brit. J. Phys. D 2, 1589 [1969].

19 W. Wonneberger, Phys. Stat. Sol. 40, 127 [1970].

20 W. Wonneberger, H. G. Reik, and A. Knoll, Phys. Letters 30 A, 46 [1969].

21 P. K. Tien, Phys. Rev. 171, 970 [1968].

22 W. H. Haydl, K. Harker, and C. F. Quate, J. Appl. Phys. 38, 4295 [1967] and W. H. HAYDL (private communication).
23 W. Wonneberger and M. Schulz, Z. Naturforsch. 26 a. 1005 [1971].

24 P. N. Butcher and N. R. OGg, Brit. J. Phys. C 3, 706 [1970].

25 D. L. Spears and R. Bray, Appl. Phys. Letters 13, 268 [1968].

26 E. G. S. PAige and H. D. Rees, Phys. Rev. Letters 16, 444 [1966].

27 J.ZuCKer and S. Zemon, Appl. Phys. Letters 9, 398 [1966].

28 W. Wettling and M. BruUn, Phys. Stat. Sol. 34, 221 [1969].

\title{
Diffusionsuntersuchungen im System Palladium-Vanadium mit Hilfe der Mikrosonde
}

\author{
Peter Lamparter, Traudl Krabichler und Siegrried Steeb \\ Max-Planck-Institut für Metallforschung, Institut für Sondermetalle, Stuttgart \\ (Z. Naturforsch. 27 a, 960-965 [1972] ; eingegangen am 25. März 1972)
}

Diffusion in the System Pd-V Investigated by Means of an Electron Microprobe

\begin{abstract}
Diffusion processes $\left(600\right.$ to $\left.1300^{\circ} \mathrm{C}\right)$ were investigated by means of quantitative electron-probemicroanalysis. The composition of the diffusion zone corresponds quite well with the phase diagram. The following phases were observed: $\mathrm{Pd}$-solid solubility, $\mathrm{Pd}_{3} \mathrm{~V}, \mathrm{Pd}_{2} \mathrm{~V}$, and $\mathrm{V}$-solid solubility. Below $900^{\circ} \mathrm{C}$ the growth of the diffusion zone is retarded; above $900^{\circ} \mathrm{C}$ the layer grows according to $d=k \cdot \sqrt{t}$. For all phases observed, according to Matano's method the coefficients of the interdiffusion were determined. The temperature dependency of these coefficients as well as that of the $k$-values follows an Arrhenius law. Thus the activation energies of the layer growth and of the interdiffusion were obtained. The determination of the intrinsic diffusion coefficients by the observation of the Kirkendall-effect is discussed.
\end{abstract}

In früheren Arbeiten wurde über Diffusionsuntersuchungen an den Systemen $\mathrm{Pd}-\mathrm{Ta}^{1}, \mathrm{Nb}-\mathrm{Pd}^{2}$ und $\mathrm{Pd}-\mathrm{Ti}^{3}$ berichtet.

Das Ziel dieser Arbeiten ist einerseits, eine Reihe von Diffusionspaarungen zu untersuchen, bei denen der eine Partner - das kubisch flächenzentrierte $\mathrm{Pd}$ - immer derselbe ist, um dadurch aus den gewonnenen Daten Aufschlüsse über den Diffusionsmechanismus solcher Paarungen zu erhalten; andererseits ergab sich die Wahl des zweiten Partners aus dem Interesse, das der Entwicklung von Schutzschichten auf Sondermetallen entgegengebracht wird.

Die Reihe der Palladium-Sondermetall-Paarungen wurde inzwischen durch Untersuchungen an den Systemen $\mathrm{Pd}-\mathrm{V}^{3}$ und $\mathrm{Pd}-\mathrm{Mo}^{4}$ ergänzt. Im folgenden soll über die Diffusionsvorgänge im System $\mathrm{Pd}-\mathrm{V}$ berichtet werden.

Sonderdruckanforderungen an Priv.-Doz. Dr. S. STEEB, MaxPlanck-Institut für Metallforschung, Institut für Sondermetalle, D-7000 Stuttgart 1, Seestraße 92.

\section{Quantitative Mikroanalyse}

Bezüglich der Auswertung der Messungen mit den Methoden der quantitativen Mikroanalyse sei auf eine frühere Arbeit ${ }^{1}$ verwiesen. Danach gilt für das Intensitätsverhältnis $I_{\text {(Probe) }}^{\mathrm{A}} / I_{\text {(Standard) }}^{\mathrm{A}}$

$$
\left(I / I_{0}\right)^{\mathrm{A}}=C_{\mathrm{G}}^{\mathrm{A}} F_{1}^{\mathrm{A}} F_{2}^{\mathrm{A}} F_{3}^{\mathrm{A}}
$$

wobei $C_{\mathrm{G}}^{\mathrm{A}}$ der Gewichtsbruchteil des Elementes A und $F_{1}^{\mathbf{A}}, F_{2}^{\mathbf{A}}, F_{3}^{\mathbf{A}}$ die Atomnummer-, Absorptionsund Fluoreszenzkorrektur bedeuten (vgl. ${ }^{1}$ ). Nach Gl. (1) wurden die zu erwartenden Intensitätsverhältnisse $\left(I / I_{0}\right)^{\mathrm{A}}$ für verschiedene $C_{\mathrm{G}}^{\mathrm{A}}$-Werte berechnet, wobei sich die durchgezogenen Linien in Abb. 1 als theoretische Eichkurven ergaben.

Zur Überprüfung der Korrekturen und damit der theoretischen Eichkurven wurden als Eichstandards Legierungen im Lichtbogenofen erschmolzen und danach homogen geglüht.

Die an diesen Eichstandards erhaltenen Meßwerte sind als Meßpunkte in Abb. 1 eingezeichnet und er- 